\title{
Contribución al conocimiento de los Anfípodos (Gammaridea) de Ibiza, islas Baleares
}

\author{
M. Ortiz* y A. Jimeno**
}

Durante la campaña oceanográfica Fauna III, organizada por el Museo Nacional de Ciencias Naturales de Madrid (CSIC) dentro del contexto del Proyecto Fauna Ibérica, se recolectaron diversas muestras cualitativas, tanto planctónicas como bentónicas, alrededor de las Islas Baleares y Columbretes. Con el presente trabajo se dan a conocer los resultados obtenidos del estudio de los Anfípodos (Gammaridea) de las muestras bentónicas de dicha campaña, correspondientes al entorno de la isla de Ibiza.

Bellan-Santini y Rufo (1998) señalan que la fauna de anfípodos del Mediterráneo, cuya superficie constituye alrededor del $2 \%$ de todos los océanos del mundo, representa aproximadamente el $10 \%$ de todas las especies marinas conocidas. Dichos autores presentan la distribución geográfica de las 48 familias, 167 géneros y 451 especies que, en aquel momento, habían sido citadas en este mar. En Mediterráneo occidental están presentes al menos 405 de las 451 especies de anfípodos citadas (Bellan-Santini y Rufo, 1998), por lo que es la zona más rica en especies de dichos crustáceos en el Mediterráneo (Bellan-Santini y Ruffo, 1998). Se ha elegido, precisamente, una área concreta dentro del Mediterráneo occidental, como es la isla de Ibiza y su entorno, de la que se disponían muestras de un gran rango batimétrico y de hábitats, a fin de valorar la representación de los anfípodos en la misma, dentro del contexto de la revisión de Bellan-Santini y Rufo (1998).

En el único trabajo previo que conocemos en el que se citan especies de este grupo de crustáceos en Ibiza es el de Ballesteros et al. (1987), que estudian las especies encontradas en 16 localidades de la mencionada isla y en 2 localidades de Formentera.
Aunque no se especifican las especies para cada una de las islas en particular, puede suponerse que la mayoría estén presentes en Ibiza, ya que es donde se recolectó más intensamente.

Los datos de las muestras de Ibiza de la campaña Fauna III en que han aparecido anfípodos (todas ellas obtenidas entre el 5 y el 10 de julio de 1994) se presentan en la Tabla 1. La lista de las especies obtenidas de dichas muestras se ofrece en la Tabla 2 y el ordenamiento taxonómico seguido es el propuesto por Ruffo (1982-1998). El material estudiado ha sido depositado íntegramente en la colección del Museo Nacional de Ciencias Naturales (CSIC), Madrid, España.

\section{Resultados}

En total se han obtenido 33 especies (3 de las cuales sólo han podido identificarse a nivel de género), pertenecientes a 14 familias y 22 géneros. Como resultado, 7 familias, 16 géneros y 26 especies se citan por vez primera en Ibiza. Las Familias mejor representadas han sido Melitidae, con 4 géneros y 7 especies, Lysianassidae, con 3 géneros y 6 especies, Aoridae, con 2 géneros y 4 especies, y Dexaminidae, con 2 géneros y 3 especies. Las especies que han mostrado una distribución más uniforme en las muestras son: Lysianassa longicornis, Ceradocus orchestipes, Leptocheirus bispinosus y Leucothoe spinicarpa. Los ejemplares de Leptocheirus sp. y Guernea sp., no han sido determinados hasta el nivel específico porque no se ajustan a las especies citadas para el área de estudio. Para alcanzar una conclusión definitiva se precisaría del estudio de material adicional. Además, Maera sp. no

* Centro de Investigaciones Marinas, Universidad de La Habana, Cuba. ortiztouzet@yahoo.com

** Dpto. Biol. Animal (Invertebrados), Facultad de Biología, Universidad de Barcelona, España. ajimeno@pie.xtec.es 
Tabla 1.- Lista de las muestras del entorno de Ibiza tomadas durante la campaña Fauna III en las que se han encontrado anfípodos. Se indica su clave, situación, profundidad y sus coordenadas geográficas. (En la clave de las muestras, una B significa que fue tomada mediante buceo y una A que fue recogida mediante arrastre).

Table 1.- List of samples collected from off Ibiza in which amphipod have been found.

\begin{tabular}{|c|c|c|c|}
\hline No .orden & Localidad & Prof. & Coordenadas geográficas \\
\hline $236 \mathrm{~B} 17$ & Al sur del faro de la isla de Tagomago & $1-4 \mathrm{~m}$ & $39^{\circ} 01.80^{\prime} \mathrm{N}-1^{\circ} 39.14^{\prime} \mathrm{E}$ \\
\hline 236 B3 & Al sur del faro de la isla de Tagomago & $28 \mathrm{~m}$ & $39^{\circ} 01.80^{\prime} \mathrm{N}-1^{\circ} 39.14^{\prime} \mathrm{E}$ \\
\hline 236 B4 & Al sur del faro de la isla de Tagomago & $17 \mathrm{~m}$ & $39^{\circ} 01.80^{\prime} \mathrm{N}-1^{\circ} 39.14^{\prime} \mathrm{E}$ \\
\hline $238 \mathrm{~A}$ & Al este del Cabo Martinet & $55-56 \mathrm{~m}$ & $38^{\circ} 54.07^{\prime}-38^{\circ} 56.29^{\prime} \mathrm{N}-1^{\circ} 31.14^{\prime}-1^{\circ} 32.77^{\prime} \mathrm{E}$ \\
\hline $239 \mathrm{~A}$ & Al sur de la isla Espardell & $58-57 \mathrm{~m}$ & $38^{\circ} 42.71^{\prime}-38^{\circ} 44.90^{\prime} \mathrm{N}-01^{\circ} 32.28^{\prime}-01^{\circ} 30.51^{\prime} \mathrm{E}$ \\
\hline 240 B4 & Punta Galera, este de la Isla Espardell & $35 \mathrm{~m}$ & $38^{\circ} 47.90^{\prime} \mathrm{N}-1^{\circ} 28.95^{\prime} \mathrm{E}$ \\
\hline 240 B6 & Punta Galera, este de la isla Espardell & $3-6 \mathrm{~m}$ & $38^{\circ} 47.90^{\prime} \mathrm{N}-1^{\circ} 28.95^{\prime} \mathrm{E}$ \\
\hline 240 B9 & Punta Galera, este de la isla Espardell & $3-5 \mathrm{~m}$ & $38^{\circ} 47.90^{\prime}-38^{\circ} 47.90^{\prime} \mathrm{N}-1^{\circ} 28.95^{\prime}-1^{\circ} 28.95^{\prime} \mathrm{E}$ \\
\hline 241 B2 & Oeste de la isla Espardell & $3-5 \mathrm{~m}$ & $38^{\circ} 47.75^{\prime} \mathrm{N}-1^{\circ} 28.32^{\prime} \mathrm{E}$ \\
\hline $241 \mathrm{~B} 3$ & Oeste de la isla Espardell & $3-5 \mathrm{~m}$ & $38^{\circ} 47.75^{\prime} \mathrm{N}-1^{\circ} 28.32^{\prime} \mathrm{E}$ \\
\hline $257 \mathrm{~A}$ & 54 millas al suroeste del Islote Vedrá & $751-761 \mathrm{~m}$ & $38^{\circ} 58.95^{\prime}-38^{\circ} 56.05^{\prime} \mathrm{N}-0^{\circ} 54.56^{\prime}-0^{\circ} 51.22^{\prime} \mathrm{E}$ \\
\hline $258 \mathrm{~B} 2$ & Norte de la isla Bleda Mayor & $6-45 \mathrm{~m}$ & $38^{\circ} 58.32^{\prime} \mathrm{N}-1^{\circ} 09.90^{\prime} \mathrm{E}$ \\
\hline $259 \mathrm{~B} 8$ & Sureste de la isla Conejera & $9 \mathrm{~m}$ & $38^{\circ} 58.68^{\prime} \mathrm{N}-1^{\circ} 13.20^{\prime} \mathrm{E}$ \\
\hline 259 B9 & Sureste de la isla Conejera & $3-4 \mathrm{~m}$ & $38^{\circ} 58.68^{\prime} \mathrm{N}-1^{\circ} 13.20^{\prime} \mathrm{E}$ \\
\hline $262 \mathrm{~B} 2$ & Punta este de Cala Eubarca & $10 \mathrm{~m}$ & $39^{\circ} 04.52^{\prime} \mathrm{N}-1^{\circ} 23.13^{\prime} \mathrm{E}$ \\
\hline 263 B2 & Punta oeste de Cala Eubarca & $44 \mathrm{~m}$ & $39^{\circ} 04.40^{\prime} \mathrm{N}-1^{\circ} 21.71^{\prime} \mathrm{E}$ \\
\hline
\end{tabular}

pudo ser identificada hasta el nivel de especie, por tratarse de un ejemplar muy incompleto.

Las especies Amphilochus neapolitanus Della Valle, 1893, Aora typica Kroyer, 1845, Maera grossimana Montagu, 1808, Hyale camptonyx (Heller, 1867), Lilljeborgia dellavallei Stebbing, 1906, Caprella acanthifera Leach, 1814, Caprella hirsuta Mayer, 1890 y Caprella liparotensis Haller, 1879, citadas por Ballesteros et al. (1987) en Ibiza y Formentera, no se han encontrado en las muestras estudiadas por nosotros. Dichos autores citaron Ampithoe ramondi Audouin, 1826 como A. vaillanti Chevreux, 1911 y Stenothoe tergestinum (Nebeski, 1881) como $S$. spinimana Chevreux, 1911, ambas especies encontradas también por nosotros.

\section{AGRADECIMIENTOS}

A la Universidad Autónoma de Madrid por haber invitado al primer autor y haberle facilitado una estancia de un mes en el Laboratorio de Zoología de la Facultad de Ciencias de la UAM. Al Dr. Angel Luque y al resto del personal del mencionado laboratorio, por su indispensable y eficiente apoyo logístico. Al Dr. José Templado del Museo Nacional de Ciencias Naturales de Madrid por habernos facilitado el material objeto de este estudio. Al Conservador de Invertebrados de dicha Institución, Miguel Villena, por su inestimable ayuda con las colecciones. El trabajo forma parte del proyecto "Fauna Ibérica VII" (DGI REN2001-1956-C17-01/GLO).

\section{Referencias}

Ballesteros, M., Castelló, J., Galles, M. \& SardÀ, R., 1987. Invertebrados alguícolas marinos de las Islas Pitiusas. Consell Insular d'Eivissa i Formentera. Conselleria d'Ecologia i Medi Ambient. Eivissa. 96 pp.

Bellan-Santini, D. \& RufFo, S., 1998. Faunistics and Zoogeography. In: S. Ruffo (ed.). The Amphipoda of the Mediterranean, Part 4. Mémoires de l'Institut Océanographique (Monaco), 13(4): 895-911.

Ruffo, S., (ed.), 1982-1998. The Amphipoda of the Mediterranean, Parts 1-4. Mémoires de l'Institute Océanographique (Monaco), 13(4): 1-959. 
Tabla 2. - Lista de las especies de anfípodos (Gammaridea) encontrados en Ibiza durante la campaña Fauna III, con indicación de las muestras donde se han encontrado. Las que ya se habían citado en la isla se señalan con un asterisco $\left(^{*}\right)$. Las demás constituyen primeras citas para Ibiza.

Table 2.- List of amphipods (Gammaridea) found in samples from off Ibiza during Fauna III Cruise. The species marked with an asterisk had been previously recorded from Ibiza.

\begin{tabular}{|c|c|}
\hline Taxa & Estaciones y observaciones \\
\hline $\begin{array}{l}\text { Familia Ampeliscidae } \\
\text { Ampelisca pilicornis Heller, } 1866\end{array}$ & 239-A, fondos detríticos con roca \\
\hline $\begin{array}{l}\text { Familia Ampithoidae } \\
\text { Ampithoe ramondi Audouin, 1826* } \\
\text { Peramphithoe spuria (Krapp-Schickel, 1978) }\end{array}$ & $\begin{array}{l}\text { 236-B17, algas esciáfilas y Pseudodistoma cyrnusense; 263-B2, coralígeno } \\
\text { 241-B2, grava y Acetabularia acetabulum }\end{array}$ \\
\hline $\begin{array}{l}\text { Familia Aoridae } \\
\text { Autonoe rubromaculatus (Ledoyer, 1973) } \\
\text { Leptocheirus bispinosus Norman, 1908 } \\
\text { Leptocheirus pectinatus (Norman, 1869) } \\
\text { Leptocheirus sp. }\end{array}$ & $\begin{array}{l}\text { 238-A, sedimento fangoso } \\
\text { 240-B6, oquedad rocosa, 263-B2, coralígeno } \\
\text { 263-B2, coralígeno } \\
\text { 258-B2 (junto al hidrozoo Aglophenia sp.) }\end{array}$ \\
\hline $\begin{array}{l}\text { Familia Colomastigidae } \\
\text { Colomastix pusilla Grube, } 1861\end{array}$ & 238-A, sedimento fangoso \\
\hline $\begin{array}{l}\text { Familia Dexaminidae } \\
\text { Dexamine spiniventris (A. Costa, 1853)* } \\
\text { Dexamine spinosa (Montagu, 1813) } \\
\text { Guernea } \text { sp. }\end{array}$ & $\begin{array}{l}\text { 259-B8, algas fotófilas; } 263-\mathrm{B} 2, \text { coralígeno } \\
\text { 240-B9, pared rocosa vertical } \\
\text { 241-B2, grava y Acetabularia acetabulum }\end{array}$ \\
\hline $\begin{array}{l}\text { Familia Eusiridae } \\
\text { Eusiroides dellavallei Chevreux, 1899* }\end{array}$ & 240-B6, oquedad rocosa \\
\hline $\begin{array}{l}\text { Familia Hyalidae } \\
\text { Hyale pontica Rathke, } 1837\end{array}$ & 236-B4, pared con Parazoanthus axinellae \\
\hline $\begin{array}{l}\text { Familia Isaeidae } \\
\text { Gammaropsis dentata } \text { Chevreux, } 1900 \\
\text { Gammaropsis } \text { cf. } \text { ulrici Krapp-Schickel y Myers, } 1979\end{array}$ & $\begin{array}{l}\text { 238-A, sedimento fangoso; 263-B2, coralígeno } \\
\text { 240-B6, oquedad rocosa; 236-B3, Mesophyllum alternans }\end{array}$ \\
\hline $\begin{array}{l}\text { Familia Ischyroceridae } \\
\text { Ericthonius brasiliensis (Dana, 1855) }\end{array}$ & 241-B2, grava y Acetabularia acetabulum \\
\hline $\begin{array}{l}\text { Familia Leucothoidae } \\
\text { Leucothoe spinicarpa (Abildgaard, 1789) }\end{array}$ & $\begin{array}{l}\text { 258-B10, bajo bloques rocosos; 238-A, sedimento fangoso; 263-B2, fango con } \\
\text { detritos }\end{array}$ \\
\hline Leucothoe venetiarum Giordani-Soika, 1950 & 238-A, sedimento fangoso \\
\hline $\begin{array}{l}\text { Famillia Lysanassidae } \\
\text { Lysianassa cesarea } \text { Ruffo, } 1987 \\
\text { Lysianassa costae Milne Edwards, 1830* } \\
\text { Lysianassa longicornis Lucas, 1849* } \\
\text { Orchomene grimaldi Chevreux, 1890 } \\
\text { Orchommene cf. simillis Chevreux, } 1912 \\
\text { Tmetonyx similis (G.O. Sars, 1891) }\end{array}$ & $\begin{array}{l}\text { 238-A, sedimento fangoso } \\
\text { 263-B2, fango con detritos } \\
\text { 238-A, sedimento; 262-B2, coralígeno; 240-B9, pared rocosa vertical; 241-B3; } \\
\text { Halopteris scoparia, 263-B2, coralígeno; 236-B3, Mesophyllum alternans } \\
\text { 257-A, en un trozo de madera } \\
\text { 238-A, sedimento fangoso } \\
\text { 238-A, sedimento fangoso }\end{array}$ \\
\hline $\begin{array}{l}\text { Familia Melitidae } \\
\text { Ceradocus orchestipes A. Costa, } 1853 \\
\text { Ceradocus semiserratus (Bate, 1862) } \\
\text { Elasmopus afinis Della Valle, } 1893 \\
\text { Elasmopus pocillimanus (Bate, 1862) } \\
\text { Elasmopus rapax A. Costa, 1853 } \\
\text { Maera inaequipes (A. Costa, 1857) }\end{array}$ & $\begin{array}{l}\text { 238-A, sedimento fangoso; 239-A, fango detrítico } \\
\text { 238-A, sedimento fangoso } \\
\text { 240-B6, oquedad rocosa } \\
\text { 259-B9, algas fotófilas } \\
\text { 259-B9, algas fotófilas } \\
\text { 262-B2, sobre Sarcotragus muscarum; 238-A, sedimento fangoso; 263-B2, coralígeno; } \\
\text { 259-B9, algas fotófilas }\end{array}$ \\
\hline $\begin{array}{l}\text { Maera sp. } \\
\text { Melita valesi S. Karaman, } 1955\end{array}$ & $\begin{array}{l}\text { 240-B4, detritos orgánicos } \\
\text { 263-B2, detritos con fango }\end{array}$ \\
\hline $\begin{array}{l}\text { Familia Phoxocephalidae } \\
\text { Harpinia ala G. Karaman, } 1987\end{array}$ & 238-A, sedimento fangoso \\
\hline $\begin{array}{l}\text { Familia Stenothoidae } \\
\text { Stenothoe tergestinum (Nebeski, 1880)* }\end{array}$ & 263-B-2, detritos con fango \\
\hline
\end{tabular}

